\title{
Edad vascular y disfunción endotelial en personas que viven a gran altura
}

Aníbal Díaz-Lazo* 1,2; Carlos Barrientos-Huamani ${ }^{1,2}$

RESUMEN

Objetivo: Determinar la relación entre edad vascular (EV) y disfunción endotelial (DE) en personas adultas que viven a más de 3000 m s.n.m.

Materiales y métodos: Se realizó estudio observacional, descriptivo, transversal en el Hospital Regional Docente Clínico Quirúrgico Daniel Alcides Carrión Huancayo-Perú, de enero a diciembre del 2015. Se calculó la EV mediante el SCORE de Framingham y la DE se evaluó con la prueba de vasodilatación mediada por flujo (VMF) en arteria braquial. Se consideró significativo $\mathrm{p}<0,05$.

Resultados: Se incluyeron 67 personas (100 \%), con edad media de 55,3 + 13,4 años (rango: 30-74), 41 (61,1\%) fueron mujeres. La EV y el valor delta fueron 57,9 + 18,3 y 2,3 + 9,3 años, respectivamente. El 53,7 \% (36 participantes) presentaron EV incrementada y 22,3 \% (15) fue mayor a 10 años. El aumento de edad, hipertensión arterial y sobrepeso fueron asociados con mayor riesgo cardiovascular. De 40 años a más se incrementa la EV en forma progresiva y es más frecuente en los varones. Las personas con DE presentaron riesgo alto $(87,5 \%)$ en comparación a los que tuvieron función endotelial normal (12,5\%) ( $p=0,007$; OR: 8,52 IC95 \% 1,7-41,4). Existe relación inversa entre la EV y la función endotelial $(r=-0.437 ; p=0.000)$. A mayor EV se incrementa la $D E$, y es mayor en los varones (Rho $=-1.0 ; p<0,010)$.

Conclusiones: Existe relación inversa entre la EV y DE. La determinación de la función endotelial podría mejorar la estratificación del riesgo cardiovascular en personas que viven a gran altura y así prevenir eventos cardiovasculares.

Palabras clave: Riesgo; Enfermedades cardiovasculares; Endotelio; Hipertensión (Fuente: DeCS BIREME).

\section{Vascular age and endothelial dysfunction in individuals living at high altitude}

\author{
ABSTRACT
}

Objective: To determine the relationship between vascular age (VA) and endothelial dysfunction (ED) in adults living at more than 3,000 m.a.s.l.

Materials and methods: An observational, descriptive, cross-sectional study was performed at the Hospital Regional Docente Clínico Quirúrgico Daniel Alcides Carrión, Huancayo - Peru, from January to December 2015. The VA was calculated using the Framingham Risk Score and the ED was evaluated with the flow-mediated dilation (FMD) test in the brachial artery. A p-value $<0.05$ was considered significant.

Results: The study included 67 individuals (100\%), whose mean age was $55.3 \pm 13.4$ years (range: $30-74)$, out of which 41 (61.1\%) were women. The VA and the delta value were $57.9 \pm 18.3$ and $2.3 \pm 9.3$ years, respectively. Fifty-three point seven percent (53.7\%) (36 participants) had an increased VA and $22.3 \%$ (15 participants) were older than age 10. Increasing age, hypertension and overweight were associated with higher cardiovascular risk. From age 40, VA progressively increases and is more common in men. Individuals with ED presented high cardiovascular risk (87.5 \%) compared to those with normal endothelial function (12.5\%) ( $p=0.007$; OR: 8.52; $95 \% \mathrm{Cl}: 1.7-41.4)$. There is an inverse relationship between VA and endothelial function $(r=-0.437 ; p=0.000)$. The higher the VA, the higher the ED, which is more frequent in men (Rho =-1.0; $\mathrm{p}<0.010)$.

Conclusions: There is an inverse relationship between VA and ED. Determining the endothelial function could improve the stratification of cardiovascular risk in individuals living at high altitude and thus prevent cardiovascular events.

Keywords: Risk; Cardiovascular diseases; Endothelium; Hypertension (Source: MeSH NLM).

1. Universidad Peruana Los Andes, Facultad de Medicina Humana. Huancayo, Perú.

2. Hospital Regional Docente Clínico Quirúrgico Daniel Alcides Carrión. Servicio de Cardiología, Laboratorio de Ecocardiografía. Huancayo, Perú.

* Autor corresponsal. 


\section{INTRODUCCIÓN}

Las enfermedades cardiovasculares son la causa más común de mortalidad en los países desarrollados (1). Así mismo, el desarrollo acelerado de la sociedad, los cambios en el estilo de vida y el incremento de la esperanza de vida han ocasionado que las enfermedades cardiovasculares pasen a ser consideradas como la principal causa de mortalidad en el mundo (2). Además, se conoce que el factor de riesgo es característica de una condición que predispone a la ocurrencia de una enfermedad ${ }^{(2,3)}$, sin embargo, se ha reportado que en personas aparentemente sanas el riesgo cardiovascular es el resultado de la interacción de múltiples factores ${ }^{(4)}$.

La población general se caracteriza por tener factores de riesgo cardiovascular lo cual ha determinado establecer guías clínicas o protocolos como el SCORE de Framingham (3), a ello se añade el cálculo de la edad vascular como un marcador de predicción de probables eventos cardiovasculares a futuro ${ }^{(5-7)}$. Existen tablas que transforman el riesgo cardiovascular absoluto en edad vascular al cuantificar en qué medida el vaso sanguíneo ha envejecido por acción de los factores de riesgo cardiovascular más allá de la edad biológica ${ }^{(5-7)}$. La conversión del riesgo absoluto en edad vascular permite comparar la edad biológica con la vascular para obtener una interpretación del riesgo absoluto al contexto de edad ${ }^{(5-8)}$. El cálculo de padecer una enfermedad cardiovascular se basa en la determinación del SCORE de Framingham, una de ellas utiliza las siguientes variables clínicas: edad, sexo, índice de masa corporal, presión arterial sistólica, diabetes mellitus y tabaquismo, se sustituye la medición del colesterol por el índice de masa corporal ${ }^{(9)}$. El concepto de edad vascular simplifica la comunicación del estado de salud de una persona ${ }^{(6-8)}$.

Por otro lado, se tiene conocimiento de que el endotelio es una fina capa de células que recubre la superficie interna de los vasos sanguíneos y se comporta como una glándula autocrina y paracrina (1); mientras que la disfunción endotelial (DE) es una actividad anormal del endotelio ${ }^{(1)}$, además la función endotelial constituye un índice integrado tanto de la carga total de riesgo cardiovascular como de la suma de factores de protección vascular en un determinado individuo ${ }^{(10)}$, en ese contexto la DE podría facilitar una mejor estratificación del riesgo cardiovascular y poner en práctica estrategias preventivas para disminuir la muerte por eventos cardiovasculares ${ }^{(2)}$. La vasodilatación mediada por flujo (VMF) medida con ecografía de la arteria braquial es una técnica no invasiva para la evaluación de la función endotelial, asimismo, la VMF de la función endotelial dependiente del óxido nítrico es un procedimiento útil y muestra una relación con la progresión de los factores de riesgos cardiovasculares convencionales ${ }^{(10,11)}$.
Aproximadamente 83 millones de individuos habitan en ciudades consideradas de elevada altitud (más de 2500 $\mathrm{m}$ s. n. m.) ${ }^{(12)}$, que son, además, ambientes geográficos naturalmente hipobáricos e hipóxicos. Esta situación es un potente activador del sistema nervioso simpático que, asociada a la presencia del estrés oxidativo, ocasiona disfunción vascular ${ }^{(13)}$. En nativos de gran altitud existe un mayor riesgo de edad vascular avanzada debido a un deterioro de la función endotelial y del músculo liso $(13,14)$ lo que podría inducir a un riesgo cardiovascular incrementado ${ }^{(15)}$.

En ese contexto, se ha diseñado un estudio con el objetivo de establecer la relación entre la disfunción endotelial y la edad vascular según el SCORE de Framingham en personas que viven a gran altura.

\section{MATERIALES Y MÉTODOS}

\section{Diseño y población de estudio}

Se diseñó un estudio observacional, descriptivo, transversal. El estudio se realizó en el Laboratorio de Ecocardiografía del Servicio de Cardiología del Hospital Regional Docente Clínico Quirúrgico Daniel Alcides Carrión de Huancayo, de enero a diciembre del 2015. El lugar donde se ha realizado la investigación es una ciudad de altura ubicada a 3270 metros sobre el nivel del mar (m s. n. m.).

La población inicial elegida estuvo conformada por adultos que han nacido y que viven en ciudades de gran altura durante toda su vida, provenientes de las zonas rurales y urbanas de la provincia de Huancayo, que fueron incorporadas al azar, previa aceptación voluntaria. La muestra se determinó mediante criterios de inclusión y exclusión. Los criterios de inclusión fueron tener de 30 a 74 años de edad, haber nacido y vivir entre los 3000 a $5000 \mathrm{~m}$ s. n. m., de ambos sexos y que aceptaron participar en el estudio. Los criterios de exclusión fueron presentar enfermedad aguda, inflamatoria, infecciosa o metabólica, tener diagnóstico de arritmia cardiaca, insuficiencia cardiaca, infarto de miocardio, accidente cerebrovascular, enfermedad vascular periférica o enfermedad psiquiátrica. El tamaño de la muestra final fue de 67 personas y se aplicó el muestreo no probabilístico por conveniencia. Los datos se registraron en un cuestionario en el que se consideraron la edad biológica y vascular, el género, los datos antropométricos, clínicos, ecográficos, y el nivel de riesgo cardiovascular según el estudio de Framingham.

\section{Variables y mediciones}

Factor de riesgo cardiovascular: Es una característica biológica o estilo de vida que cuenta la probabilidad de padecer o de morir a causa de una enfermedad cardiovascular en aquellos individuos que lo presenten ${ }^{(14,15)}$. 
Diabetes mellitus: Cuando se encontró un nivel de glicemia $>126 \mathrm{mg} / \mathrm{dl}$ en examen realizado en ayunas 0 tener diagnóstico previo de diabetes mellitus ${ }^{(16)}$.

Hipertensión arterial: Fue considerado cuando los valores de presión arterial (PA) obtenidos fueron $>140 / 90 \mathrm{mmHg}$ al realizar dos mediciones con tensiómetro de mercurio, en reposo, con paciente en posición sentada, o tuvieron el diagnóstico de hipertensión arterial con valores normales y que estuvieron recibiendo tratamiento ${ }^{(17)}$.

Índice de masa corporal (IMC) ${ }^{(17)}$ : Se determinó con la medición del peso corporal (kilogramos), dividido por la altura al cuadrado (metros ${ }^{2}$ ). Se considera sobrepeso un IMC entre 25 a $29 \mathrm{kgs} / \mathrm{m}^{2}$ y obesidad el IMC $>30 \mathrm{kgs} / \mathrm{m}^{2}$.

Dislipidemia: Fue considerada cuando presentó, al menos, un valor alterado del perfil lipídico con niveles de colesterol sérico en ayunas $>200 \mathrm{mg} / \mathrm{dl}$, colesterol $\mathrm{LDL}>$ a $100 \mathrm{mg} / \mathrm{dl}$, colesterol $\mathrm{HDL}<50 \mathrm{mg} / \mathrm{dl}$ en mujeres $\mathrm{y}<40$ $\mathrm{mg} / \mathrm{dl}$ en varones, o triglicéridos $>150 \mathrm{mg} / \mathrm{dl}^{(18)}$.

Tabaquismo: Haber fumado por lo menos una vez durante el último año ${ }^{(19)}$.

Sedentarismo: Si reportó que no realiza actividad física por más de 30 minutos por día ${ }^{(20)}$.

Nivel de riesgo cardiovascular: Se aplicó el modelo de riesgo de Framingham, que fue considerado de la siguiente manera: Bajo riesgo (cuando el riesgo de un evento cardiovascular en 10 años es menor al $10 \%$ ), riesgo intermedio (cuando el riesgo de un evento cardiovascular a 10 años está comprendido entre 10 a $20 \%$ ) y alto riesgo (cuando el riesgo de un evento cardiovascular a 10 años es mayor al $20 \%{ }^{(5)}$.

Edad vascular: Es una forma de transformar el riesgo absoluto en otro concepto más entendible por el paciente ${ }^{(5-7,21)}$ y consiste en la edad que tendría una persona del mismo sexo con el mismo nivel de riesgo cardiovascular estimado, pero con todos los factores de riesgo en niveles normales ${ }^{(5-7,21)}$.

Variación delta: Es la diferencia entre la edad vascular y la edad cronológica o biológica (7).

Disfunción endotelial: Se consideró como diagnóstico de $\mathrm{DE}$, la dilatación posthiperemia de la arteria braquial por la presencia de menos del $10 \%$ de dilatación del diámetro de la arteria con relación al diámetro basal (11).

Ciudad de gran altura: Localidad ubicada entre los 3000 a 5000 m s. n. m ${ }^{(22)}$.

Procedimientos

El estudio ecográfico de la arteria braquial fue realizado por un médico cardiólogo con amplia experiencia en estudios ultrasonográficos vasculares. La función endotelial se evaluó por dilatación dependiente del endotelio (DDE) con la prueba de hiperemia reactiva o dilatación mediada por flujo sanguíneo (DMF) estudiada por ultrasonografía, que constituye el método no invasivo de referencia para evaluar la DE ${ }^{(10,11,23)}$. Para realizar el procedimiento se empleó ecógrafo Philips con transductor lineal de $10 \mathrm{MHz}$, la medición se realizó con el paciente en posición supina, en reposo, en el brazo izquierdo, en las mañanas.

La arteria braquial fue identificada en secciones longitudinales y localizadas a $5-10 \mathrm{~cm}$ del pliegue del codo. Las imágenes ultrasonográficas fueron tomadas en reposo y durante la hiperemia reactiva. El diámetro interno de la arteria braquial fue medido al final de la diástole y la velocidad del flujo sanguíneo fue evaluada con ecografía doppler pulsado con muestra tomada en el centro de la arteria braquial. El esfignomanómetro se insufló en el antebrazo izquierdo hasta $200 \mathrm{mmHg}$ durante 5 minutos, después de realizar el desinflado, fue nuevamente medido el diámetro de la arteria braquial entre los 60 a 90 segundos posteriores a la relajación del manguito de medición ${ }^{(10,11)}$. Se calculó el porcentaje de variación de la dilatación mediada por flujo (DMF) con la siguiente fórmula: IDMF = [(diámetro posthiperemiadiámetro basal) $\times 100]$ /diámetro basal $(9,10)$.

\section{Análisis estadístico}

Se realizó el análisis estadístico descriptivo con frecuencias y medias, el análisis inferencial con $\mathrm{X} 2$, $\mathrm{t}$ de Student, coeficiente de relación de Rho Spearman, regresión lineal simple y múltiple con SPSS V22. Se consideró significativo un $\mathrm{p}<0.05$.

\section{Consideraciones éticas}

El estudio fue autorizado por el Comité de Ética de la Oficina de Apoyo a la Docencia e Investigación del Hospital Regional Docente Clínico Quirúrgico Daniel Alcides Carrión de Huancayo. Se obtuvo el consentimiento informado de cada participante. Los datos recolectados durante el estudio se utilizaron solo para fines de investigación y se manejaron como confidenciales.

\section{RESULTADOS}

En el estudio se incluyeron $67(100 \%)$ personas, 26 $(35,4 \%)$ fueron varones y $41(64,6 \%)$ mujeres, el promedio de edad fue 55, $3 \pm 13,4$ años (rango: $30-74$ ). En la tabla 1 se muestra que a medida que se incrementa el promedio de edad, la presión arterial sistólica y diastólica se aumenta el nivel de riesgo cardiovascular. Además, la frecuencia de los factores de riesgo cardiovascular tradicionales tales como la diabetes mellitus, hipertensión arterial y el sobrepeso fueron asociados a un nivel de riesgo alto. 
Tabla 1. Riesgo cardiovascular según score de Framingham y características clínicas epidemiológicas

\begin{tabular}{|c|c|c|c|}
\hline Variables & $\begin{array}{l}\text { Riesgo bajo } \\
\qquad N=33\end{array}$ & $\begin{array}{l}\text { Riesgo medio } \\
\qquad N=18\end{array}$ & $\begin{array}{l}\text { Riesgo alto } \\
n=16\end{array}$ \\
\hline Edad (años), $\mathrm{X} \pm \mathrm{DS}$ & $46,4 \pm 12,3$ & $62,1 \pm 6,7$ & $66,6 \pm 7,3$ \\
\hline Varones, n (\%) & $4(12,1)$ & $10(55)$. & $12(75,0)$ \\
\hline Mujeres, n (\%) & $29(87,9)$ & $8(44,5)$ & $4(25,0)$ \\
\hline Índice de masa corporal $\left(\mathrm{kg} / \mathrm{m}^{2}\right), \mathrm{X} \pm \mathrm{DS}$ & $26,0 \pm 5,0$ & $26,1 \pm 3,4$ & $25,1 \pm 3,4$ \\
\hline Presión arterial sistólica, $\mathrm{X} \pm \mathrm{DS}$ & $107,4 \pm 11,5$ & $124,4 \pm 8,3$ & $138,1 \pm 27,8$ \\
\hline Presión arterial diastólica, $\mathrm{X} \pm \mathrm{DS}$ & $70,3 \pm 11,2$ & $81,9 \pm 10,0$ & $83,1 \pm 14,7$ \\
\hline Frecuencia cardiaca (lat $x \min$ ), $X \pm D S$ & $73,9 \pm 14,2$ & $71,4 \pm 11,4$ & $70,2 \pm 12,6$ \\
\hline Saturación $\mathrm{O}_{2}(\%), \mathrm{X} \pm \mathrm{DS}$ & $92,9 \pm 2,6$ & $92,6 \pm 3,4$ & $92,5 \pm 4,2$ \\
\hline Hipertensión arterial, n (\%) & $2(6,0)$ & $8(44,4)$ & $4(25,0)$ \\
\hline Sobrepeso, n (\%) & $10(30,3)$ & $6(33,3)$ & $6(37,0)$ \\
\hline Obesidad, n (\%) & $7(21,2)$ & $4(7,0)$ & $2(12,5)$ \\
\hline Diabetes mellitus, $\mathrm{n}(\%)$ & $2(6,0)$ & $2(11,1)$ & $3(18,7)$ \\
\hline Dislipidemia, n (\%) & $0(0,0)$ & $5(27,7)$ & $0(0,0)$ \\
\hline Sedentarismo, n (\%) & $4(12,1)$ & $1(5,5)$ & $1(6,2)$ \\
\hline
\end{tabular}

Fuente: Cuestionario de recolección de datos. Edad vascular y disfunción endotelial en personas que viven a gran altura. 2015

De la muestra de 67 (100\%) personas incluidas en el estudio, $23,9 \%$ (16) presentaron riesgo cardiovascular alto, 26,9\% (18) riesgo medio y 46,1\% (33) riesgo bajo. En la figura 1 se muestra que $70,7 \%$ (29) de las mujeres presentaron riesgo cardiovascular bajo en comparación al $15 \%$ (4) de los varones $(p=0,0001)$. El 46,1 \% (12) de varones presentaron riesgo cardiovascular alto, cifra alta en relación al 9,8\% (4) de las mujeres $(p=0,0016)$. El 23,9\% (16), 26,9\% (18) y 46,1\% (33)

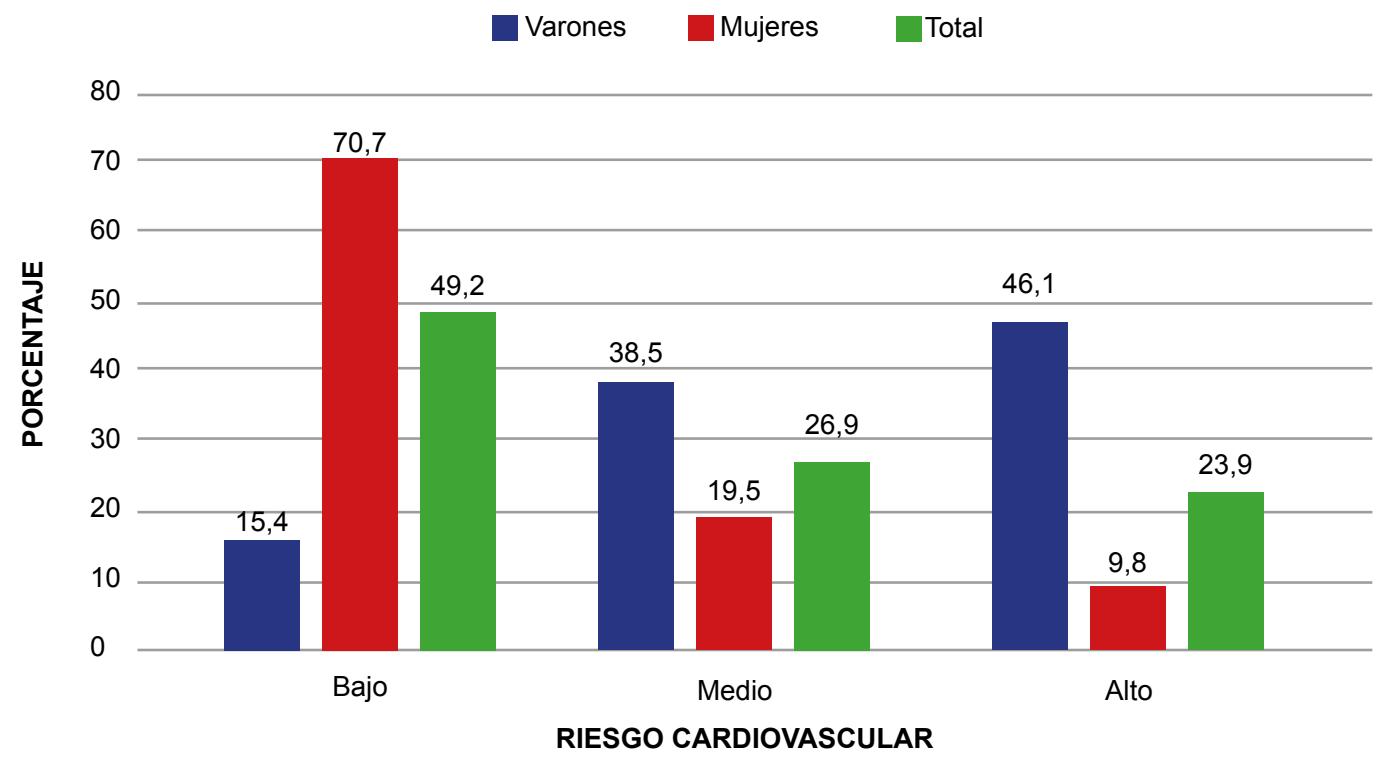

Fuente: Cuestionario de recolección de datos. Edad vascular y disfunción endotelial en personas que viven a gran altura. 2015

Figura 1. Riesgo cardiovascular global a 10 años según SCORE de Framingham y género 
El $53,7 \%$ (36) de personas presentaron una EV incrementada en relación a la EB, $22(32,8 \%)$ personas por encima de los 6 años y 15 (22,3\%) personas más de 10 años. La EB y EV fueron mayores en los varones $(59,6$ $\pm 12,1$ y $63,1 \pm 14,5)$ en comparación a las mujeres $(52,8$ $\pm 13,554,5 \pm 19,5)$. El incremento promedio de la EV fue de 3,6 años en los varones y de 1,5 años, en las mujeres. La EB y EV total fue de $55,4 \pm 13,4$ y $57,8 \pm 18,3$ años, respectivamente, y se observa un incremento global de 2,4 años.

En la tabla 2 se muestra la edad biológica y vascular según grupo etario. Se observa que a partir de la cuarta década de la vida se incrementa la edad biológica, así como también, aumenta la edad vascular, incremento que es mucho mayor desde los 60 años.

Tabla 2. Edad biológica y vascular según grupo etario

\begin{tabular}{|c|c|c|c|}
\hline Grupo etario (años) & $\begin{array}{c}\text { Edad biológica,X } \\
\text { + DS }\end{array}$ & $\begin{array}{c}\text { Edad vascular, } X \\
+ \text { DS }\end{array}$ & Delta, X + DS \\
\hline $30-39$ & $33,0 \pm 3,0$ & $31,3 \pm 6,7$ & $-1,6 \pm 5,4$ \\
\hline $40-49$ & $45,5 \pm 3,4$ & $47,5 \pm 12,3$ & $2,0 \pm 10,2$ \\
\hline $50-59$ & $53,5 \pm 2,7$ & $56,2 \pm 12,2$ & $2,9 \pm 11,7$ \\
\hline $60-74$ & $67,5 \pm 4,1$ & $71,2 \pm 10,4$ & $3,6 \pm 8,4$ \\
\hline
\end{tabular}

Fuente: Cuestionario de recolección de datos. Edad vascular y disfunción endotelial en personas que viven a gran altura. 2015

Para evaluar la asociación entre la DE y EV se distribuyó a la población en dos grupos. En uno, los que presentaron DE $\left(n_{1}\right)$, que fueron $37(55,2 \%)$; y en el segundo grupo los que no tuvieron diagnóstico de $\operatorname{DE}\left(\mathrm{n}_{2}\right)$ que fueron $30(44,8 \%)$. En la tabla 3 , se muestra que las personas con $D E$ incrementaron su edad biológica a vascular en promedio 5,4 años $(p=0,065)$, a diferencia de las personas con función endotelial normal donde la edad vascular estuvo disminuida en promedio 1,4 años en comparación a la edad biológica $(p=0.754)$. Asimismo, el promedio de edad biológica es mucho menor en las personas con función endotelial preservada o normal en comparación a las personas con DE.

Tabla 3. Edad biológica y vascular en personas con función endotelial normal y disfunción

\begin{tabular}{|lccc|}
\multicolumn{1}{c|}{ Variable } & $\begin{array}{c}\text { Edad biológica, } \\
\text { X+ DS }\end{array}$ & $\begin{array}{c}\text { Edad vascular, } \mathbf{X} \\
+ \text { DS }\end{array}$ & \begin{tabular}{c} 
Delta, $\mathbf{X}+$ DS \\
\hline Disfunción endotelial
\end{tabular} \\
\hline Función endotelial normal & $61,2+10,3$ & $66,7+14,3$ & $5,4+-9,3$ \\
\hline
\end{tabular}

Fuente: Cuestionario de recolección de datos. Edad vascular y disfunción endotelial en personas que viven a gran altura. 2015

En la figura 2 se observa que las personas con DE presentaron riesgo cardiovascular alto $(87,5 \%)$ a 10 años, según el SCORE de Framingham, en comparación a los que tuvieron función endotelial normal $(12,5 \%)(p=0,007)$, a diferencia del grupo que reportó riesgo cardiovascular bajo, cuya mayor frecuencia fue asociada a las personas con función endotelial normal $(63,6 \%)$, en comparación al grupo con DE $(36,4 \%)(p=0,002)$. Asimismo, se muestra que a medida que se incrementa el nivel de riesgo cardiovascular se aumenta la frecuencia de DE, la que se relaciona, medianamente, de forma inversa con el grupo de personas que presentaron función endotelial normal, lo cual no fue significativo ( $R$ de Spearman $=-0.5 ; p=$ $0,667)$. 


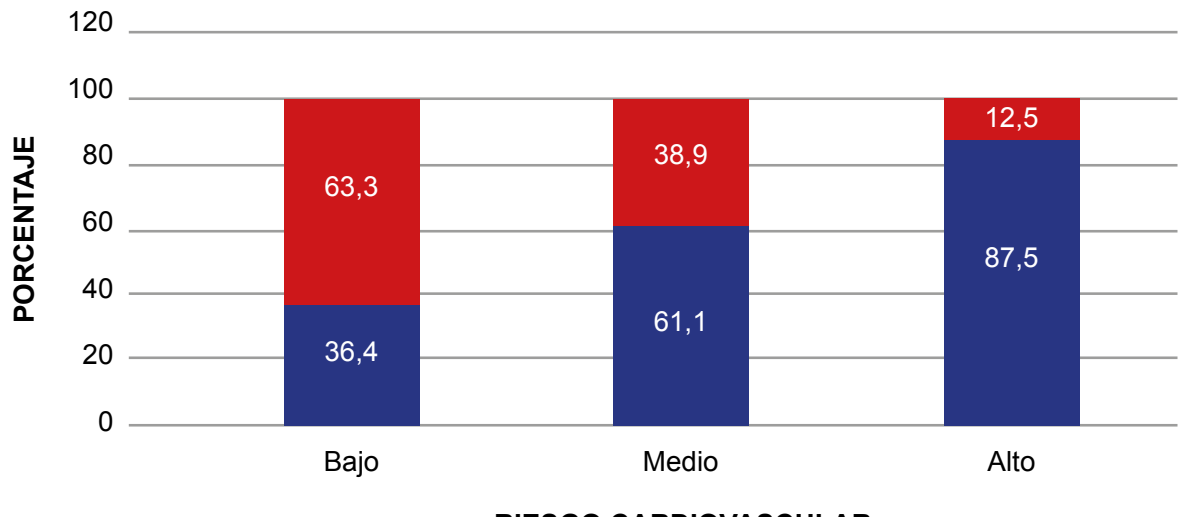

RIESGO CARDIOVASCULAR

Fuente: Cuestionario de recolección de datos. Edad vascular y disfunción endotelial en personas que viven a gran altura. 2015

Figura 2. Riesgo cardiovascular global a 10 años según SCORE de Framingham en personas con disfunción endotelial

En la figura 3 se observa un incremento progresivo del nivel de riesgo en los varones con disfunción endotelial, la misma que se relaciona de forma inversa con el nivel de riesgo de las mujeres ( $R$ de Spearman $=-1,0 ; p<0,010$ )

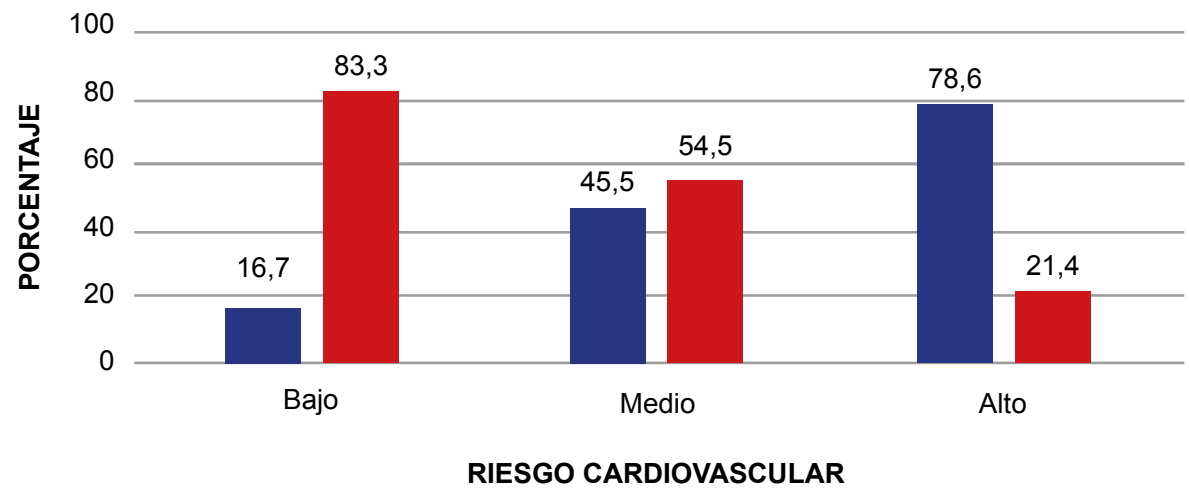

Fuente: Cuestionario de recolección de datos. Edad vascular y disfunción endotelial en personas que viven a gran altura. 2015

Figura 3. Nivel de riesgo cardiovascular en personas con disfunción endotelial según género

Al aplicarse regresión lineal simple se encontró que existe relación inversa entre la EV y la función endotelial $(r=-0,437 ; p=0,000)$, y entre la EB y la función endotelial $(r=-0,360 ; p=0,003)$. Al aplicarse la regresión lineal múltiple se encontró que el incremento de la edad vascular se relaciona de forma inversa significativamente con el deterioro de la función endotelial $(r=-0,392 ; p=$ 0,031 ) y no se encontró significancia con la EB (Figura 4). 


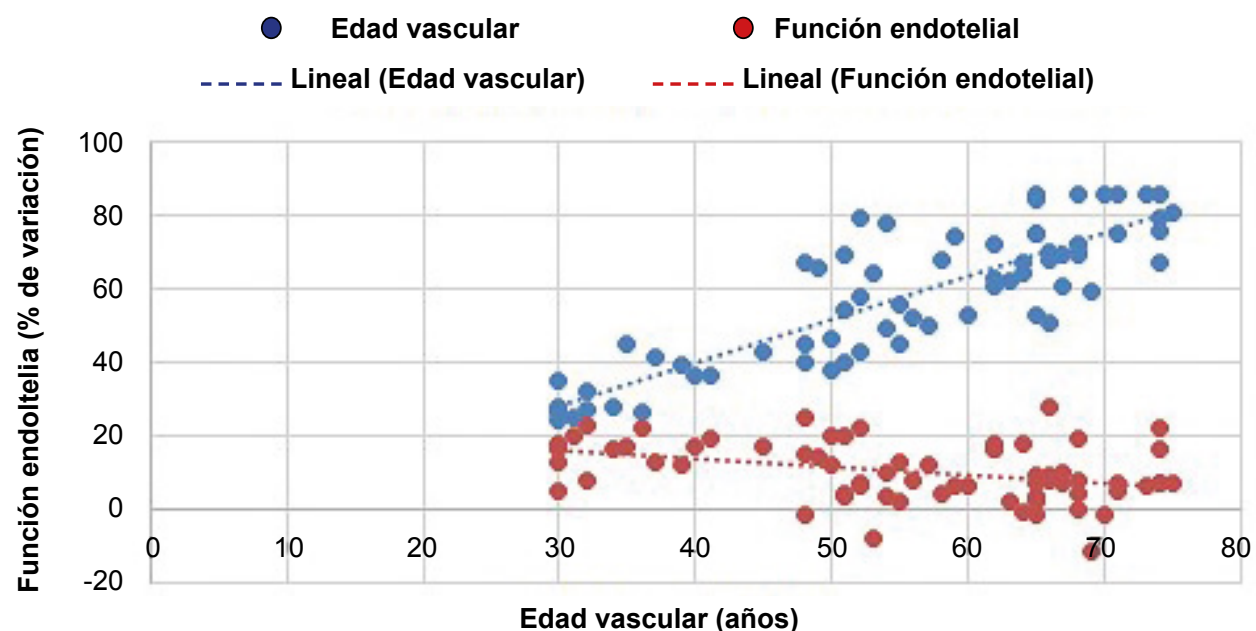

Fuente: Cuestionario de recolección de datos. Edad vascular y disfunción endotelial en personas que viven a gran altura. 2015

Figura 4. Edad vascular y función endotelial en personas que viven a gran altura

En la figura 5 se observa que, de cada tres personas con edad vascular incrementada, dos presentaron DE y solo en un tercio se encontró $\mathrm{DE}$ con edad vascular normal $(p=0,002)$.

Disfunción endotelial

Función endotelial normal

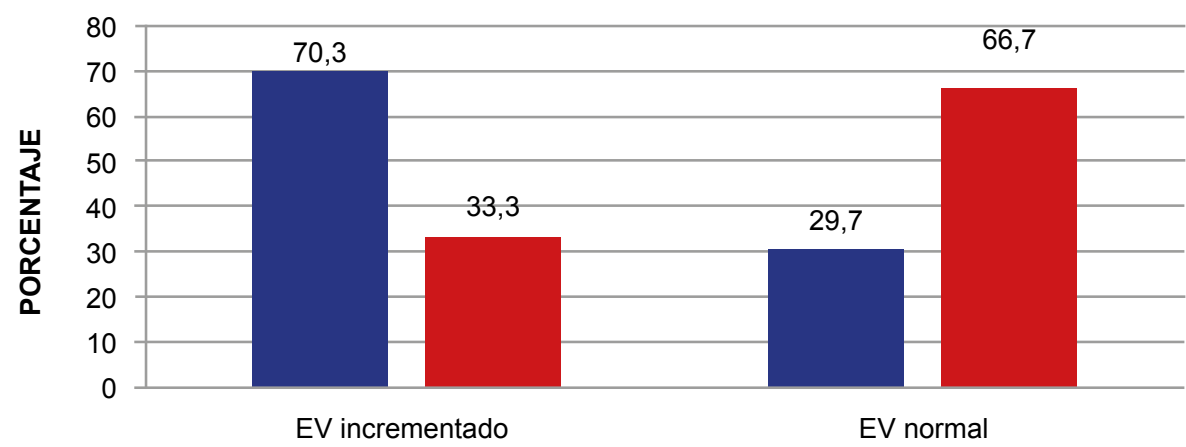

EDAD VASCULAR

Fuente: Cuestionario de recolección de datos. Edad vascular y disfunción endotelial en personas que viven a gran altura. 2015

Figura 5. Edad vascular y disfunción endotelial en personas que viven a gran altura

\section{DISCUSIÓN}

En el mundo, la mortalidad debido a enfermedades cardiovasculares se ha incrementado en un tercio entre 1990 y el 2010; para el año 2015 se había previsto que una de cada tres muertes sería ocasionada por enfermedad cardiovascular ${ }^{(24)}$.

Los factores de riesgo actúan como iniciadores, promotores, potenciadores y precipitadores en el proceso 
de la enfermedad cardiovascular $y$, generalmente, no se presenta un único factor de riesgo, sino que un gran número de pacientes muestran varios, que interactúan unos con otros, lo que genera una potenciación en el resultado final, como se observa cuando existe una combinación de hipercolesterolemia, hipertensión arterial y tabaquismo, que aumenta hasta en 16 veces el riesgo cardiovascular ${ }^{(25)}$.

Para una valoración integral de la salud de las personas es fundamental determinar el riesgo cardiovascular y así establecer tratamientos óptimos para una atención diferenciada según el nivel de riesgo. En ese contexto, son útiles las escalas de medida del riesgo cardiovascular, como la que se deriva del estudio de Framingham, el proyecto SCORE (Systemic Coronary Risk Evaluation 2003) que se aplica en Europa, el QRISK en Reino Unido o el estudio PROCAM para la población alemana, que tratan de establecer el mejor puntaje de riesgo para sus respectivas poblaciones con las características propias de sus regiones (26-29).

Se ha descrito en todas las tablas de score de estratificación de riesgo cardiovascular que la edad es un factor muy importante al determinar un mayor puntaje $y$, por lo tanto, incrementar riesgo ${ }^{(5,25)}$. El puntaje de Framingham que se ha aplicado utiliza seis factores de riesgo como variables no modificables, la edad y el género; así mismo, las variables susceptibles de ser modificables como presión arterial sistólica, tabaquismo, diabetes mellitus, colesterol total y colesterol HDL (este último es sustituido por índice de masa corporal) ${ }^{(5,25)}$. Un análisis crítico que se realiza a las tablas de medición del riesgo cardiovascular es que este cálculo es una probabilidad poblacional que se aplica a individuos concretos, por lo que no siempre se identifican a los sujetos que van a presentar el evento cardiovascular (21). En consecuencia, cuando se calcula el riesgo cardiovascular hay que considerar a la población de origen, el evento cardiovascular que se mide y el horizonte temporal en que se da que generalmente, es a 10 años (21-30).

En la búsqueda de la valoración del riesgo cardiovascular integral, se estableció la edad vascular que representa la edad hipotética del sistema cardiovascular que podría ser una alternativa para precisar, aún más, el riesgo cardiovascular de una persona ${ }^{(6,21,31)}$. Al respecto, Cuende et al. (2013) señalan que el principal problema que se ha reportado en personas jóvenes con niveles elevados de los factores de riesgo cardiovascular es que presentan un riesgo cardiovascular absoluto bajo, pero con el concepto de edad vascular se estima un riesgo cardiovascular relativo alto, lo que amerita implementar las medidas terapéuticas ${ }^{(31)}$.

La puntuación de Framingham promedio según Herrera et al. ${ }^{(32)}$, fue $15,1+-7,17$, en nuestro estudio fue $11,73+$ -
9,29. Ruiz et al. (2013) ${ }^{(25)}$, en una investigación realizada en 25 ciudades del Perú reportó, para el año 2004, una prevalencia de riesgo cardiovascular absoluto bajo $(63,3 \%)$, medio $(18,8 \%)$ y alto $(17,8 \%)$; y para el año 2010 , encontró $59,8 \%$ de riesgo bajo, $19,5 \%$ de riesgo medio y $20,7 \%$ en riesgo alto. El estudio de Mayta-Calderón et al.(33) (2015), en pacientes hospitalizados en un servicio de medicina interna, encontró que $48,3 \%$ estaba en riesgo bajo, $31,1 \%$ en riesgo medio y $20,6 \%$ en riesgo alto. En nuestra serie encontramos que 16 pacientes $(23,9 \%)$ presentaron riesgo cardiovascular alto y 18 participantes $(26,9 \%)$, riesgo medio, estos porcentajes son elevados en comparación a lo reportado por estudios previos ${ }^{(25,33)}$.

Se ha descrito que los factores de riesgo cardiovascular asociados a riesgo alto y medio fueron el sexo masculino, la edad avanzada, la diabetes mellitus y el tabaquismo ${ }^{(33)}$; en nuestra serie no se encontró asociación con el tabaquismo, pero sí con la edad avanzada, el sexo masculino y la diabetes. En la literatura plantea que, a medida que aumentan los valores de la presión arterial sistólica y diastólica, se incrementa el nivel de riesgo ${ }^{(33)}$. En nuestro estudio también encontramos un incremento del nivel de riesgo cardiovascular con el aumento de la presión arterial.

En la ciudad de Huancayo, durante el año 2004, se reporta una edad biológica promedio de 47 años y edad vascular de 52 años; mientras que para el año 2010, se reporta 50 y 55 años para la edad biológica y vascular, respectivamente ${ }^{(3,25)}$. En nuestro estudio la edad biológica y vascular fueron más altas.

En la investigación de Masson et al. (7) en una población española, los hombres tenían un valor delta de edad mayor que las mujeres ( 6 años). El estudio de Mayta-Calderón et al. (33) reportó un valor delta de 6,9 años, que es mayor en los hombres ( 9 años), que en las mujeres ( 4,5 años), además encontró delta - 0,8 años en pacientes de riesgo bajo ; de 10,5 en el grupo de riesgo medio, y 16 años en el de riesgo alto ${ }^{(33)}$. Nosotros encontramos valores delta menores en comparación a ciudades localizadas a menor altura, y con una mayor diferencia en los varones.

La edad vascular está relacionada con el riesgo cardiovascular global, lo que determina los años que el paciente puede ganar o perder según controle sus factores de riesgo cardiovascular (2). Así, la edad vascular de un individuo es igual a la edad que tendría una persona con igual riesgo cardiovascular global, pero con todos los factores de riesgo dentro de los valores normales (2). Es decir, cuantifica en qué medida la arteria ha envejecido por efecto de los factores de riesgo más allá de la edad biológica. La conversión de riesgo absoluto en edad vascular permite comparar la edad biológica con la vascular para obtener una interpretación del riesgo 
cardiovascular relativo en el contexto de la edad, y si la diferencia es elevada debe recibir tratamiento de inmediato, independientemente si el valor absoluto sea mayor o menor, porque significa que tiene un pobre estilo de vida ${ }^{(1,6,7,25)}$. Si la edad vascular es menor o mayor que la edad cronológica, una persona podría ser reclasificada en otra categoría de riesgo ${ }^{(6,7)}$.

En consecuencia, la edad vascular nos indica la edad que le corresponde a las arterias de un individuo; si este valor se conoce, podría mejorarse la adherencia de los pacientes al tratamiento. Se describe en estudios previos, como el de Mayta-Calderón et al. ${ }^{(33)}$, que los valores encontrados para la edad biológica y vascular son similares a los hallazgos de nuestra serie. Además, en varones se reporta riesgo alto en $75,7 \%$, riesgo mediano en $55 \%$ y riesgo bajo en $31,3 \%$. En nuestro estudio también hallamos porcentajes similares con riesgo alto $(75 \%$ ) y riesgo mediano ( $55 \%$ ).

Mayta-Calderón et al. ${ }^{(33)}$, encontraron en su serie de pacientes, que el $68,9 \%$ presentó una edad mayor que la edad cronológica. González (34) (2015) en su estudio con pacientes hipertensos reportó un incremento de edad vascular de 1 año a más en 94,8 \% de los casos; de 6 a más años, en $82,7 \%$; y de 11 a más en $56,8 \%$. La edad cronológica es menor en comparación a la edad vascular, tanto en varones como en mujeres, hallazgos similares son reportados en otros estudios ${ }^{(33,34)}$. En nuestra casuística también encontramos casos con mayor aumento de la edad vascular en comparación a la edad biológica, sin embargo, solo el $53 \%$ mostraron este aumento.

La hipertensión arterial aumenta de 2 a 3 veces el riesgo de todas las enfermedades arterioescleróticas, en especial, la enfermedad cerebro-vascular y la insuficiencia cardiaca ${ }^{(5)}$. La hipertensión arterial acelera y aumenta la remodelación vascular relacionada con el incremento de la edad y esta puede impactar sobre la severidad del daño vascular en la hipertensión lo que indica la interacción entre la edad biológica y la elevación de la presión arterial (1). Gonzales (34) en un estudio de 58 personas hipertensas encontró $15,5 \%$ de factor de riesgo cardiovascular, en hombres fue $17,3 \%$ y en las mujeres $8,5 \%$. El $24 \%$ presentaron riesgo alto y el $47 \%$, riesgo medio. Hallazgos similares a nuestro estudio, en el que la frecuencia es mayor en varones en comparación a las mujeres, la misma que podría ser explicada por el comienzo tardío de la enfermedad cardiaca en la mujer debido, probablemente, a la acción protectora de los estrógenos ${ }^{(34)}$.

Por otro lado, a la DE se ha relacionado con los factores de riesgo coronario, de forma que la vasodilatación mediada por flujo sanguíneo está disminuida en estadios tempranos de la enfermedad cardiovascular en pacientes con hipertensión arterial, hipercolesterolemia, fumadores y diabéticos ${ }^{(35)}$. Calderon-Gerstein et al., en su investigación realizada a $3250 \mathrm{~m}$ s. n. m. reporta DE en $100 \%$ de diabéticos, $68,8 \%$ de obesos, y en $55 \%$ de hipertensos (36). Otro estudio al mismo nivel de altitud encontró DE en $82,4 \%, 71,5 \%$ y $50 \%$ de individuos hipertensos, diabéticos y obesos respectivamente ${ }^{(36)}$.

La determinación de la DE puede ayudar a definir mejor la edad vascular del paciente, lo cual permite establecer un tratamiento óptimo y adecuado pronóstico de los pacientes. Un estudio previo realizado en 72 pacientes reporta una frecuencia de 45,8 \% de DE (32). Encontramos porcentaje muy similar $(55,2 \%)$ en nuestra serie. Una investigación de casos y controles realizada en una ciudad de altura reporta una frecuencia de $57,9 \%$ de DE en pacientes con factores de riesgo cardiovascular (casos) y de $46,5 \%$ de $\mathrm{DE}$ en los controles ${ }^{\left({ }^{37}\right)}$. En nuestro estudio, hallamos DE en personas con FRCV modificables en $64,4 \%$ y DE en personas sin FRCV en $36,6 \%$. Es probable que la edad asociada a factores ambientales como la hipoxia crónica podría estar relacionada a la elevada prevalencia de DE en pobladores de altura sin FRCV tradicionales.

Según el nivel de estratificación de riesgo cardiovascular Herrera et al. ${ }^{(32)}$ encontraron que la frecuencia de DE en el grupo compuesto por pacientes de alto riesgo, riesgo medio y riesgo bajo fue de $75 \%, 55,6 \%$ y $12 \%$, hallazgos muy similares a nuestra casuística. Yao et al. (2014) reportaron en un estudio de 1083 pacientes entre 30 a 80 años, que la vasodilatación mediada por flujo disminuye con el incremento del SCORE de Framingham en ambos sexos, (en varones $R=-0,308 ; p<0,001$; y para mujeres: $R=-0.577 ; p<0,001)^{(38)}$. Campuzano et al., en un estudio de 52 personas con una media de 55,77+- 14,96 años, sin distribución por sexos encontró que la función endotelial es peor en pacientes con factores de riesgo, pero sin enfermedad ateroesclerótica clínica, que en pacientes sin ellos (35), al analizar los resultados obtuvo que la vasodilatación mediada por flujo fue casi 4 veces mayor en el grupo de pacientes sin factores de riesgo en comparación a los que presentaron al menos un factor de riesgo ${ }^{(36)}$. En nuestra serie encontramos hallazgos similares, se objetiva que a mayor número de factores de riesgo cardiovascular es mayor la disfunción endotelial, también se encontró que la hipertensión arterial fue casi siete veces más frecuente en personas con DE en comparación a los portadores de función endotelial normal $(\mathrm{OR}=6,72$; IC95 \% = 1,37-32,99; $p=0,009)$. No se encontró asociación significativa con los otros factores de riesgo cardiovascular.

Existen estudios en poblaciones tibetanas, pero estas difieren genéticamente por su evolución histórica con la población andina; por lo tanto, al comparar los cambios adaptativos vasculares de ambos grupos podrían diferir a pesar de habitar a igual altitud, por lo que, al interpretar los resultados se deben considerar todas las variables 
involucradas ${ }^{(13)}$. En nuestro estudio se encontró una correlación inversa entre la disfunción endotelial y la edad vascular, a mayor edad vascular existe menor variación del diámetro de la arteria braquial, por lo tanto, mayor disfunción endotelial.

La importancia de esta investigación radica en proponer una estrategia destinada a precisar el nivel de riesgo cardiovascular que añade el estudio ultrasonográfico de la arteria braquial a la medición de la función endotelial.

Una limitación del estudio es el escaso número de participantes, así como la no equivalencia entre varones y mujeres. Esto no permite generalizar los resultados, por lo que es recomendable realizar más investigaciones.

En ese contexto, un propósito es la búsqueda del mejoramiento continuo de la calidad de vida de la población para lo cual debe, primeramente, realizarse un reconocimiento inicial del problema, y aplicar, para ello, la puntuación de Framingham, determinar la edad vascular para que, posteriormente, las personas con riesgo cardiovascular mediano y alto se sometan al estudio de la ultrasonografía de la arteria braquial para determinar la disfunción endotelial, lo que mejoraría la precisión del nivel de riesgo cardiovascular. Por último, debemos sugerir que en las personas con delta de más de 5 años de EV es recomendable realizar estudio ultrasonográfico de la arteria braquial para mejorar la estratificación de riesgo cardiovascular.

En conclusión, existe relación inversa entre la EV y la función endotelial $(r=-0,437 ; p=0,000)$ en personas que habitan a gran altura.

Es elevada la frecuencia de DE en personas con riesgo cardiovascular alto $(87 \%)$ y medio $(61 \%)$ y la función endotelial normal estuvo asociado a riesgo bajo (63\%).

Más de la mitad de las personas ( $53 \%$ ) presentaron una edad vascular incrementada en relación a la edad biológica, y el delta promedio de la EV fue mayor en las personas con DE (5 años) en comparación a las personas cuantificadas solo con el puntaje de Framingham ( 2 años).

El incremento de la edad y de los niveles de presión arterial se asocian con niveles de riesgo cardiovascular medio y alto en forma progresiva.

\section{REFERENCIAS BIBLIOGRÁFICAS}

1. Harvey A, Montezano AC, Touyz RM. Vascular biology of ageing-Implication in hypertension. J Mol Cell Cardiol. 2015 Jun;83:112-21.

2. Achiong Alemañy $M$, Achiong Estupiñán $F$, Achiong Alemañy F, Afonso de León JA, Álvarez Escobar MC, Suárez Merino M.
Riesgo cardiovascular global y edad vascular: herramientas claves en la prevención de enfermedades cardiovasculares. Rev Med Electrón. 2016 Mar-Abr;38(2):211-6.

3. Ruíz Mori E, Segura Vega L, Agusti Campos R. Uso del score de Framingham como indicador de los factores de riesgo de las enfermedades cardiovasculares en la población peruana. Rev Per Card. 2012 Set-Dic;38(3):1-19

4. Perk J, De Backer G, Gohlke H, Graham I, Reiner Z, Verschuren $M$, et al. Guía Europea sobre prevención de la Enfermedad Cardiovascular en la Práctica Clínica (versión 2012). Rev Esp Cardiol. 2012;65(10):937.e1-e66.

5. D’Agostino RB, Vasan RS, Pencina MJ, Wolf PA, Cobain M, Massaro JM, et al. General cardiovascular risk profile for use in primary care: the Framingham Heart Study. Circulation. 2008 Feb 12;117(6):743-53.

6. Cuende JI, Cuende N, Calaveras-Lagartos J. How to calculate vascular age with the SCORE project scales: a new method of cardiovascular risk evaluation. Eur Heart J. 2010 Oct;31(19): 2351-8.

7. Masson W, Siniawski D, Toledo G, Vita T, Fernández G, Del Castillo $S$, et al. Estimación de la "edad vascular" basada en el índice de masa corporal en una población en prevención primaria. Asociación con la aterosclerosis carotídea subclínica. Med Clin. 2013 Mar 16;140(6):255-9.

8. Leal Herrera U, Espinoza de Leal M. La edad vascular: Un nuevo concepto de fácil compresión. Salus. 2015 MayAgo, 19(2):5-6.

9. Gaziano TA, Young CR, Fitzmaurice G, Atwood S, Gaziano JM. Laboratory-based versus non-laboratory-based method for assessment of cardiovascular disease risk: the NHANES I Follow-up Study cohort. Lancet. 2008 Mar 15; 371(9616):92331

10. Arrebola-Moreno AL, Laclaustra M, Kaski JC. Evaluación no invasiva de la función endotelial en la práctica clínica. Rev Esp Cardiol. 2012; 65(1):80-90.

11. Celermajer DS, Sorensen KE, Gooch VM, Spiegelhalter DJ, Miller OI, Sullivan ID, et al. Non-invasive detection of endothelial dysfunction in children and adults at risk of atherosclerosis. Lancet. 1992 Nov 7;340(8828):1111-5

12. Narváez-Guerra 0 , Herrera-Enríquez K, Medina - Lezama J, Chirinos JA. Systemic Hypertension at high altitude. Hypertension. 2018 Sep;72(3):567-78.

13. Lewis NC, Bailey DM, Dumanoir GR, Messinger L, Lucas SJ, Cotter JD, et al. Conduit artery structure and function in lowlanders and native highlanders: relationships with oxidative stress and role of sympathoexcitation. J Physiol. 2014 Mar 1;592(5):1009-24.

14. Chobanian AV, Bakris GL, Black HR, Cushman WC, Green LA, Izzo JL Jr, et al. Seventh Report of the Joint National Committee on Prevention, Detection, Evaluation, and Treatment of High Blood Pressure. Hypertension. 2003 Dec; 42(6):1206-52.

15. Lobos Bejarano JM, Brotons Cuixart M. Factores de riesgo cardiovascular y atención primaria: evaluación e intervención. Aten Prim. 2011 Dic;43(12):625-84.

16. American Diabetes Association. Diagnosis and classification of Diabetes Mellitus. Diabetes Care. 2010 Jan;33(Suppl 1):S62-9.

17. Berrington de Gonzales A, Hartge P, Cerhan JR, Flint AJ, Hannan L, Maclnnis RJ, et al. Body Mass Index and Mortality among 1.46 million white adults. N Engln J Med. 2010 Dec 2;363 (23). :2211-9. 
18. Pasternak RC. Report of the Adult Treatment Panel III: the 2001 National Cholesterol Education Program guidelines on the detection, evaluation and treatment of elevated cholesterol in adults. Cardiol Clin. 2003 Aug;21(3):393-8.

19. Moreno Moreno LP. Prevalencia de los principales factores de riesgo de enfermedad cardiovascular y riesgo cardiovascular en pacientes con hipertensión arterial que asisten a una IPS en Bogotá y algunos municipios de Cundinamarca [Tesis]. Bogotá: Pontificia Universidad Javeriana; 2009. pp. 1-92.

20. Cabrera de León A, Rodríguez-Pérez MC, Rodríguez-Benjumeda LM, Ania-Lafuente B, Brito-Díaz B, Muros de Fuentes $M$, et al. Sedentary Lifestyle: Physical activity duration versus percentage of energy expenditure. Rev Esp Cardiol. 2007 Mar;60(3):244-50.

21. Cuende JI. La edad vascular frente al riesgo cardiovascular: aclarando conceptos. Rev Esp Cardiol. 2016 Mar;69(3):243-6.

22. Todo vertical-Guías de montañas. [modificar por Internet] Disponible en: http://www.todovertical.com/articulo/6/ aclimatacion-adaptacion-a-la-altitud-prevencion-del-malagudo-de-montana

23. Cruz Hernández J, Licea Puig ME, Hernández García P, Yanes Quesada M, Salvato Dueñas A. Disfunción endotelial y diabetes mellitus. Rev Cubana Endocrinol. 2012;23(2):166-85.

24. Mahmood SS, Levy D, Vasan RS, Wang TJ. The Framingham Heart Study and the epidemiology of cardiovascular diseases: a historical perspective. Lancet. 2014 Mar 15;383(9921):999-1008.

25. Ruíz Mori E. Riesgo y Prevención Cardiovascular. 1a ed. Lima: Unigraph Editores; 2014.

26. Alcocer LA, Lozada O, Fanghanel G, Sánchez-Reyes L, Campos-Franco E. Estratificación del riesgo cardiovascular global. Comparación de los métodos Framingham y SCORE en población mexicana del estudio PRIT. Circ. 2011;79(2):168-74.

27. Baena Díez JM, Del Val García JL, Salas Gaetgens LH, Sánchez Pérez R, Altes Vaques E, Deixens Martínez B, et al. Comparación de los modelos SCORE y REGICOR para el cálculo del Riesgo Cardiovascular en sujetos sin enfermedad cardiovascular atendidos en un centro de salud de Barcelona. Rev Esp Salud Publica. 2005 Jul-Ago;79(4):543-64.

28. Conroy RM, Pyorala K, Fitzgerald AP, Sans S, Menotti A, De Backer $G$, et al. Estimation of ten-year risk of fatal cardiovascular disease in Europe: the SCORE Project. Eur Heart J. 2003 Jun;24(11):987-1003.

29. Assmann G, Cullen P, Schulte H. Simple scoring scheme for calculating the risk of acute coronary events based on the 10-year follow-up of the prospective cardiovascular Munster (PROCAM) study. Circulation. 2002 Jan 22;105(3):310-5

30. Cuende JI, Saltijeral A, Estrada V, Pérez de Isla L. Vascular age calculation and equivalent risk factors in HIV-infected patients obtained from the D:A:D risk equation. Eur J Prev Cardiol. 2016 Nov;23(17):1903-8.

31. Cuende JI. Edad vascular derivada del SCORE y Guía Europea sobre prevención de la enfermedad cardiovascular en la práctica clínica (versión 2012). Rev Esp Cardiol. 2013;66(3): 241.

32. Herrera Tanaka V, Chavez Ayala C. Relación entre reactividad

flujo de arteria braquial y SCORE de riesgo de Framingham como marcador de disfunción endotelial. Rev Per Cardiol. 2006;34(3):152-8.

33. Mayta Calderón JC, Morales Moreno AM, Cárdenas Rojas AD, Mogollón Lavi JA, Armas Rodríguez V, Neyra Arismendiz L, et al. Determinación de riesgo cardiovascular y edad vascular según SCORE de Framingham en pacientes del Hospital Nacional Arzobispo Loayza. Horiz Med. 2015 Abr-Jun;15(2):27-34.

34. González Ariztimuño E. Valoración del riesgo cardiovascular y edad vascular en personas con hipertensión primaria [Tesis]. España: Universidad del País Vasco UPV/EHU; 2014/2015. pp. 1-26.

35. Campuzano R, Moya JL, García-Lledó A, Salido L, Guzmán G, Tomas JP, et al. Asociación de la disfunción endotelial y el grosor mediointimal carotídeo con los factores de riesgo coronario en pacientes sin evidencia clínica de aterosclerosis. Rev Esp Cardiol. 2003;56(6):546-54.

36. Calderón - Gerstein WS, López-Peña A, Macha-Ramirez R, Bruno-Huamán A, Espejo-Ramos R, Vílchez-Bravo S, et al. Endothelial dysfunction assessment by flow-mediated dilation in a high-altitude population. Vasc Health Risk Manag. 2017 Nov 21;13:421-6.

37. Díaz A. Factores de riesgo cardiovascular y disfunción endotelial en adultos que viven a gran altura. Acta Méd Peru. 2016 Oct-Dic;33(4):289-95.

38. Yao F, Liu Y, Liu D, Wu S, Lin H, Fan R, et al. Sex differences between vascular endothelial function and carotid intimamedia thickness by Framingham Risk Score. J Ultrasound Med. 2014 Feb;33(2):281-6.

Fuentes de financiamiento:

Este artículo ha sido financiado por los autores.

Conflictos de interés:

Los autores declaran no tener ningún conflicto de interés.

Correspondencia:

Aníbal Díaz Lazo

Dirección: Calle Ricardo Palma № 553 Lima 31, Perú.

Teléfono: 51-4810838

Correo electrónico: andiaz55@hotmail.com

Recibido: 27 de setiembre de 2018 . Evaluado: 12 de diciembre de 2018 Aprobado: 01 de febrero de 2019

(c) La revista. Publicado por Universidad de San Martín de Porres, Perú.

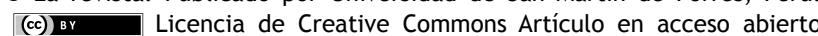
bajo términos de Licencia Creative Commons Atribución 4.0 Internacional. (http://creativecommons.org/licenses/by/4.0/)

ORCID iDs

Anibal Diaz Lazo

Carlos Barrientos-Huamani https://orcid.org/0000-0002-9282-9435 https://orcid.org/0000-0003-4652-8655 